\title{
A HYPERFINITE FACTOR WHICH IS NOT AN INJECTIVE C*-ALGEBRA
}

\author{
Kazuyuki Saitô \\ 2-7-5 Yoshinari, Aoba-ku \\ Sendai 989-3205 \\ Japan \\ (yk.saito@beige.plala.or.jp)
}

\author{
J.D. Maitland Wright \\ University of Aberdeen \\ Aberdeen, Scotland \\ (j.d.m.wright@abdn.ac.uk); \\ Christ Church \\ University of Oxford \\ (maitland.wright@chch.ox.ac.uk)
}

July 2019

\begin{abstract}
We exhibit a wild monotone complete $\mathrm{C}^{*}$-algebra which is a hyperfinite factor but is not an injective $\mathrm{C}^{*}$-algebra.
\end{abstract}

\section{INTRODUCTION}

A von Neumann factor is hyperfinite if it is generated by an increasing sequence of finite dimensional matrix algebras. It is straightforward to show that such a factor is an injective $C^{*}$-algebra. See [14], Chapter XVI Corollary 1.8 or [8].

A much harder result, due to Connes [2], is the converse. If a (small) von Neumann factor is an injective $C^{*}$-algebra then it is hyperfinite.

Let $M$ be a $\mathrm{C}^{*}$-algebra with self-adjoint part $M_{s a}$. If each upper bounded, upward directed subset of $M_{s a}$ has a least upper bound then $M$ is said to be monotone complete. (For a detailed account of monotone complete $\mathrm{C}^{*}$-algebras, see [11]). Every von Neumann algebra is monotone complete but the converse is false. However it seemed plausible to conjecture that if a monotone complete factor is hyperfinite, in a suitable sense, then it is injective. If this could be established for a particular monotone complete factor ([13] and [4]) then this would imply a positive solution of the Marzewski problem [18]. (See also [15, 1]). More precisely, Banach-Tarski paradoxical decompositions can be achieved using pieces which are measurable with respect to the Baire Property. In an impressive tour de force, Dougherty and Foreman [3] obtained this conclusion by completely different methods. This made the injectivity of the Takenouchi-Dyer factor seem even more plausible. But this is false.

We exhibit a monotone complete $\mathrm{C}^{*}$-algebra which is a hyperfinite factor but is not injective. See Theorem 10. 
This can be deduced by applying some delicate and intricate arguments of Hjorth and Kechris [7]. Here we use a different approach which can be applied more generally. We give an argument which we hope readers will find transparent; key tools used include Theorem 3.4 [12] and our Lemma 1, (on properties of the Free Group, $\mathbb{F}_{2}$, and equivariant linear maps).

\section{PRELIMINARIES AND BACKGROUND}

A commutative unital $\mathrm{C}^{*}$-algebra $C(E)$ is monotone complete if and only if the compact Hausdorff space $E$ is extremally disconnected. In other words, the closure of each open subset of $E$ is clopen.

Let $T$ be an Hausdorff topological space. Then $T$ is said to be perfect if it has no isolated points. Furthermore $T$ is a Polish space if it is homeomorphic to a complete separable metric space. Let $B(T)$ be the (commutative) algebra of bounded complex-valued Borel functions on a perfect Polish space $T$. Let $M(T)$ be the ideal of those $f$ in $B(T)$ for which $\{t \in T: f(t) \neq 0\}$ is meagre. Then $B(T) / M(T)$ is isomorphic to $B(\mathbb{R}) / M(\mathbb{R})$. This quotient algebra is known as the Dixmier algebra. It is monotone complete; we denote it by $C(S)$. The compact extremally disconnected space $S$ has no isolated points but has a countable dense subset. $C(S)$ is not a von Neumann algebra because it has no normal states [11].

Let $B$ be any commutative unital $\mathrm{C}^{*}$-algebra. Then $B \cong C(K)$, where $K$ is compact Hausdorff. Let $\theta$ be a homeomorphism of $K$ onto itself. Define $h_{\theta}$ on $C(K)$ by $h_{\theta}(f)=f \circ \theta$. Then $\theta \rightarrow h_{\theta}$ is a group anti-isomorphism from Homeo $(K)$, (the group of all homeomorphisms of $K$ onto $K$ ), onto Auto $(C(K))$, (the group of all $*$-automomorphisms of $C(K)$ ). It follows that $\theta \rightarrow h_{\theta}^{-1}$ is an isomorphism of $\operatorname{Homeo}(K)$ onto Auto $(C(K))$.

Throughout this paper, $G$ will be a countable group. Let $X$ be a perfect Polish space or a dense $G_{\delta}$ subset of $S$. (So the Baire category theorem is valid for $X$.) Then an action of $G$ on $X$ is a homomorphism $\alpha$ from $G$ into the group of homeomorphisms of $X$ onto $X$. Much of what follows is valid for more general extremally disconnected spaces than $S$ but we shall focus on the Dixmier algebra, $C(S)$.

Let $Z$ be a $G$-invariant subset of $X$. Then the action $\alpha$ is said to be topologically free over $Z$, if whenever $g \in G$ and $g$ is not the identity, then $\alpha_{g}$ has no fxed points in $Z$.

The action $\alpha$ is said to be (generically) free, if whenever $g \in G$ and $g$ is not the identity, then the set of fixed points of $\alpha_{g}$ is a closed nowhere dense subset of $X$. When this occurs, there is a, $G$-invariant, dense $G_{\delta}$ subset $Z \subset X$, such that the action $\alpha$ is topologically free over $Z$.

The action $\alpha$ is said to be (generically) ergodic if, for some $x_{0} \in X$, the orbit $\left\{\alpha_{g}\left(x_{0}\right): g \in G\right\}$ is dense in $X$. When this occurs, it follows from Lemma 1.1 [12] that there is a, $G$-invariant, dense $G_{\delta}$ subset $Y \subset X$ such that for every $y \in Y$, the orbit $\left\{\alpha_{g}(y): g \in G\right\}$ is dense in $Y$.

Let $A$ be any commutative monotone complete $\mathrm{C}^{*}$-algebra. Let $\Gamma$ be a 
countable group of $*$-automorphisms of $A$. So $A \cong C(E)$, where $E$ is compact Hausdorff and extremally disconected.

We recall that a $*$-automorphism, $h$ of $A$ is said to be properly outer if there does not exist a nonzero projection $e$ in $A$ such that the restriction of $h$ to $e A$ is the identity map. The action of $\Gamma$ on $A$ is said to be free if every element of $\Gamma$, other than the identity, is a properly outer automorphism. The action of $\Gamma$ on $A$ is said to be ergodic if, given a projection $p, g(p)=p$ for all $g$ implies that $p=0$ or $p=1$.

Let $\Gamma$ be a countable infinite group of $*$-automorphisms of $C(S)$ which acts freely and ergodically. There is a corresponding monotone cross-product $\mathrm{C}^{*}$ algebra, $M(C(S), \Gamma)$. (For monotone cross-products see $[9,10,5,11,12,13]$ ). This algebra is a TypeIII factor which contains the Dixmier algebra as a maximal abelian $*$-subalgebra and hence is not a von Neumann algebra $[9,10]$. See also $[16,17]$. By Theorem 3.4 [12] this algebra is unique. Every free, ergodic action of an infinite countable group $G$ on $C(S)$ gives rise to the same factor. By [12] this algebra is hyperfinite. It corresponds, modulo meagre sets, to a canonical hyperfinite Borel equivalence relation on a perfect Polish space. We shall show that it is not injective.

The strategy is as follows. Let $A=C(S)$ and let $B$ be a commutative monotone complete $\mathrm{C}^{*}$-algebra with $A$ a subalgebra of $B$. Let $G$ be a (countable) group of $*$-automorphisms of $B$ which are also automorphisms of $A$. Then, by Proposition 2.3 [18], if $M(A, G)$ is injective there exists a $G$-equivariant, positive linear projection $\Phi$ of $B$ onto $A$. On putting $B=\ell^{\infty}(G)$ and taking $G$ to be the free group on two generators, it will follow from our technical lemma, Lemma 1, that $\Phi$ vanishes on $B$. But this is impossible since $\Phi(1)=1$. So this contradiction shows that the hyperfinite factor, $M(C(S), G)$ is not injective.

\section{EQUIVARIANT LINEAR MAPS OVER THE FREE GROUP}

Let $G$ be a countable group. Let $\ell^{\infty}(G)$ be the commutative $C^{*}$-algebra of all complex valued, bounded sequences on $G$. Let $\gamma^{g}$ be the automorphism of $\ell^{\infty}(G)$ defined by

$$
\left(\gamma^{g} f\right)(h)=f\left(g^{-1} h\right) \text { for each } g, h \in G \text { and } f \in \ell^{\infty}(G) .
$$

Let $\mathbb{F}_{2}$ be the free group on two generators $a$ and $b$. Let $\langle a\rangle$ be the subgroup of $\mathbb{F}_{2}$ generated by $a$ (so $<a>\cong \mathbb{Z}$ ). Let $e$ be the empty word. For each reduced word $x$, let $W(x)$ be the set of reduced words beginning with $x$.

There are many ways known of obtaining paradoxical decompositions of $\mathbb{F}_{2}$. Wagon [15] gives a lucid and elegant exposition.See in particular his Theorem 4.2 and the related discussions. For our purposes, it is convenient to use the following decomposition.

For each $i \in \mathbb{Z}$ we put

$$
H_{i}=\bigcup\left\{W\left(a^{i} b^{i_{1}}\right): i_{1} \neq 0\right\} .
$$


Then we have $H_{i} \cap H_{j}=\varnothing$ if $i \neq j$. Moreover, we have

$$
\mathbb{F}_{2}=<a>\cup \bigcup_{i \in \mathbb{Z}} H_{i}
$$

where each $\left.H_{i} \cap<a\right\rangle=\varnothing$.

Observe that the map $\lambda_{a}: x \mapsto a x\left(x \in \mathbb{F}_{2}\right)$ satisfies

$$
\lambda_{a} H_{i}=H_{i+1} \text { for each } i \in \mathbb{Z} .
$$

Also the map $\lambda_{b}: y \mapsto b y\left(y \in \mathbb{F}_{2}\right)$ satisfies $\lambda_{b} H_{i} \subset H_{0}(i \neq 0)$ and $\lambda_{b}<a>\subset$ $H_{0}$.

Observe that, for each $E \subset \mathbb{F}_{2}, \chi_{E} \in \ell^{\infty}\left(\mathbb{F}_{2}\right)$ and for each $g \in \mathbb{F}_{2}$,

$$
\left(\gamma^{g} \chi_{E}\right)(h)=\chi_{E}\left(g^{-1} h\right)=\chi_{\lambda_{g} E}(h) \text { for each } h .
$$

Let $T$ be a Hausdorff topological space with no isolated points. Let $g \rightarrow \alpha_{g}$ be a group homomorphism from a countable group $G$ into $\mathrm{Homeo}(T)$, the group of homeomorphisms of $T$. Let $t_{0}$ be a point such that $\left\{\alpha_{g}\left(t_{0}\right): g \in G\right\}$ is a dense orbit. Also suppose the orbit is free, that is, the map

$$
g \rightarrow \alpha_{g}\left(t_{0}\right)
$$

is a bijection from $G$ onto the orbit.

Let $\alpha^{g}(f)(t)=f\left(\alpha_{g^{-1}} t\right)$. Then $g \rightarrow \alpha^{g}$ is a group isomorphism of $G$ into $\operatorname{Aut}\left(C_{B}(T)\right)$, the group of all automorphisms of $C_{B}(T)$, the $C^{*}$-algebra of all bounded, complex-valued continuous functions on $T$.

There is a natural embedding $J$ of $C_{B}(T)$ into $\ell^{\infty}(G)$ given by

$$
J f=\left(f\left(\alpha_{h}\left(t_{0}\right)\right)\right)_{h \in G} .
$$

Then $\gamma^{g}$ restricted to $J\left[C_{B}(T)\right]$ coincides with the automorphism of $J\left[C_{B}(T)\right]$ induced by $\alpha^{g}$.

Indeed, for $f \in C_{B}(T)$ and $g, h \in G$, we have

$\left(\gamma^{g} J f\right)(h)=(J f)\left(g^{-1} h\right)=f\left(\alpha_{g^{-1} h} t_{0}\right)=f\left(\alpha_{g^{-1}} \alpha_{h} t_{0}\right)=\left(\alpha^{g}(f)\right)\left(\alpha_{h} t_{0}\right)=J \alpha^{g}(f)(h)$,

which implies $\gamma^{g} J=J \alpha^{g}$ for all $g \in G$.

Now specialise by putting $G=\mathbb{F}_{2}$ and require $T$ to be compact and extremally disconnected. Then $C_{B}(T)=C(T)$. Extremal disconnectedness implies that whenever $D$ is a dense subset of $T$ then the Stone-Čech compactification of $D$ can be identified with $T$. (See Theorem 6.2.7 in [11]) Each element of $J\left[C_{B}(T)\right]$ restricts to a bounded continuous function defined on the orbit $\left(\alpha_{h}\left(t_{0}\right)\right)_{h \in G}$, equipped with the relative topology induced by $T$. Conversely, by Stone-Cech, each such function is the restriction of a unique continuous function in $C(T)$.

Lemma 1 Let $\phi$ be a positive linear map from $\ell^{\infty}\left(F_{2}\right)$ to $J[C(T)]$ such that

$$
\gamma^{g} \circ \phi=\phi \circ \gamma^{g} \text { for each } g \in \mathbb{F}_{2} \text {. }
$$


Further suppose that the sub orbit $\left\{\alpha_{a^{n}} t_{0}: n \in \mathbb{Z}\right\}=<a>\left[t_{0}\right]$ is dense in $T$. We recall that $T$ has no isolated points. Then the linear map $\phi$ is identically zero.

Proof. We have

$$
\gamma^{a} \phi\left(\chi_{H_{i}}\right)=\phi\left(\gamma^{a} \chi_{H_{i}}\right)=\phi\left(\chi_{\lambda_{a} H_{i}}\right)=\phi\left(\chi_{H_{i+1}}\right) \text { for each } i \in \mathbb{Z} .
$$

This implies that

$$
\left(\gamma^{a}\right)^{n} \phi\left(\chi_{H_{i}}\right)=\phi\left(\chi_{H_{i+n}}\right) \text { for all } i, n \in \mathbb{Z} .
$$

We have

$$
\begin{aligned}
\phi\left(\chi_{\mathbb{F}_{2}}\right)\left(t_{0}\right) & \geq \phi\left(\sum_{i=-m}^{m}\left(\chi_{H_{i}}\right)\right)\left(t_{0}\right)=\sum_{i=-m}^{m} \phi\left(\chi_{H_{i}}\right)\left(t_{0}\right) \\
& \geq \sum_{i=-m}^{m}\left(\gamma^{a}\right)^{i}\left(\phi\left(\chi_{H_{0}}\right)\right)\left(t_{0}\right) \text { for each } m \in \mathbb{N} .
\end{aligned}
$$

So $\left(\gamma^{a}\right)^{n}\left(\phi\left(\chi_{H_{0}}\right)\right)\left(t_{0}\right) \rightarrow 0$ as $|n| \rightarrow \infty$. But

$$
\left(\gamma^{a}\right)^{n}\left(\phi\left(\chi_{H_{0}}\right)\right)\left(t_{0}\right)=\phi\left(\chi_{H_{0}}\right)\left(a^{-n} t_{0}\right) \text { for each } n \in \mathbb{Z} \text {. }
$$

So $\phi\left(\chi_{H_{0}}\right)\left(a^{-n} t_{0}\right) \rightarrow 0$ as $|n| \rightarrow \infty$. So, for any given positive number $\epsilon$, there is an $m_{0} \in \mathbb{N}$ such that

$$
\left|\left(\gamma^{a}\right)^{n}\left(\phi\left(\chi_{H_{0}}\right)\right)\left(t_{0}\right)\right|=\left|\phi\left(\chi_{H_{0}}\right)\left(a^{-n} t_{0}\right)\right|<\epsilon \text { for all } n \text { with }|n| \geq m_{0} .
$$

Since $T$ has no isolated points, $\left\{a^{n}\left(t_{0}\right):|n| \geq m_{0}\right\}$ is dense in $T$. It follows that $\left|\phi\left(\chi_{H_{0}}\right)\left(a^{n}\left(t_{0}\right)\right)\right| \leq \epsilon$ for all $n$. By applying the Stone-Čech compactification theorem, $\phi\left(\chi_{H_{0}}\right)$ has a unique extension to a continuous function on $T$ which is norm bounded by $\epsilon$ for all $\epsilon>0$. So $\phi\left(\chi_{H_{0}}\right)=0$. By positivity, if $S \subset H_{0}$ then $\phi\left(\chi_{S}\right)=0$. Also $0=\gamma^{g} \phi\left(\chi_{S}\right)=\phi\left(\gamma^{g}\left(\chi_{S}\right)\right)=\phi\left(\chi_{\lambda_{g} S}\right)$. We now recall $\lambda_{b} H_{i} \subset H_{0}(i \neq 0)$. So

$$
\lambda_{b}\left(\bigcup_{i \neq 0} H_{i}\right) \subset H_{0} .
$$

So $\phi$ vanishes on the characteristic function $\chi_{\cup_{i \neq 0} H_{i}}$. Similarly, $\lambda_{b}<a>\subset H_{0}$ implies that $\phi$ vanishes on the characteristic function of $\langle a\rangle=\left\{a^{n}: n \in \mathbb{Z}\right\}$. Hence $\phi$ is identically zero.

\section{CONSTRUCTING FREE GROUP ACTIONS WITH DENSE SUB-ORBITS}

In this section we construct actions of the free group, $\mathbb{F}_{2}$, that we shall need when applying Lemma 1. 
First we shall consider the Cantor space over $\mathbb{F}_{2}$ with the shift action induced by $\mathbb{F}_{2}$. See [6]. We equip, $2^{\mathbb{F}_{2}}$ with the product topology. Then it is a compact Hausdorff space which is a perfect Polish space, that is, it is homeomorphic to a complete seperable metric space with no isolated points. (In fact it is homeomorphic to the Cantor set.) The action of $\mathbb{F}_{2}$ on $2^{\mathbb{F}_{2}}$ is defined, for each $g \in \mathbb{F}_{2}$ by

$$
\left(\alpha_{g}(x)\right)(h)=x\left(g^{-1} h\right) \text { for } x \in 2^{\mathbb{F}_{2}} \text { and } h \in \mathbb{F}_{2} .
$$

When there is no risk of ambiguity, we put $g \cdot x=\alpha_{g}(x)$. So

$$
(g \cdot x)(h)=x\left(g^{-1} h\right) \text { for } x \in 2^{\mathbb{F}_{2}} \text { and } h \in \mathbb{F}_{2} .
$$

Let us recall that for each $g \in \mathbb{F}_{2}$, this map $2^{\mathbb{F}_{2}} \ni x \mapsto g \cdot x \in 2^{\mathbb{F}_{2}}$ is a homeomorphism from $2^{\mathbb{F}_{2}}$ onto itself and the action $\mathbb{F}_{2} \ni g \mapsto \alpha_{g} \in \operatorname{Homeo}\left(2^{\mathbb{F}_{2}}\right)$ is a group homomorphism such that for $g_{1}, g_{2} \in \mathbb{F}_{2}$ and $x \in 2^{\mathbb{F}_{2}}$,

$\left(\left(g_{1} g_{2}\right) \cdot x\right)(h)=x\left(g_{2}^{-1} g_{1}^{-1} h\right)=\left(g_{2} \cdot x\right)\left(g_{1}^{-1} h\right)=\left[g_{1} \cdot\left(g_{2} \cdot x\right)\right](h)$ for each $h \in \mathbb{F}_{2}$.

Take $g \in \mathbb{F}_{2} \backslash\{e\}$ and let

$$
F(g)=\left\{x \in 2^{\mathbb{F}_{2}}: g \cdot x=x\right\} .
$$

Since $\alpha_{g}$ is a homeomorphism, $F(g)$ is closed. So its compliment

$$
O(g)=\left\{x \in 2^{\mathbb{F}_{2}}: g \cdot x \neq x\right\}
$$

is open. Since $\mathbb{F}_{2}$ acts on $2^{\mathbb{F}_{2}}$ as the shift action, $O(g) \neq \emptyset$.

Moreover, we have $y \in F\left(h g h^{-1}\right)$ iff $h g h^{-1} \cdot y=y$ iff $g \cdot\left(h^{-1} \cdot y\right)=h^{-1} \cdot y$ iff $h^{-1} \cdot y \in F(g)$ iff $y \in h \cdot F(g)$. So

$$
h \cdot O(g)=O\left(h g h^{-1}\right) \text { for each } h \in \mathbb{F}_{2} .
$$

We claim $O(g)$ is dense. To do this, take a non-empty open subset $O$. We may assume that $O$ is a basic open subset and so we may assume

$$
O=\left\{x \in 2^{\mathbb{F}_{2}}: x(h)=\iota_{h} \text { if } h \in F\right\}
$$

for some non-empty finite subset $F$ of $\mathbb{F}_{2}$ and $\iota_{h} \in\{0,1\}$ for $h \in F$. Since $F \cup g F$ is finite, there is $h_{0} \in \mathbb{F}_{2}$ such that $h_{0} \notin F$ and $g^{-1} h_{0} \notin F$. Let

$$
x(h)= \begin{cases}0 & \text { if } h=h_{0} \\ 1 & \text { if } h=g^{-1} h_{0} \\ \iota_{h} & \text { if } h \in F \\ 0 & \text { if } h \in \mathbb{F}_{2} \backslash\left(F \cup\left\{h_{0}, g^{-1} h_{0}\right\}\right) .\end{cases}
$$

Clearly $x \in O$. On the other hand, we have

$$
(g \cdot x)\left(h_{0}\right)=x\left(g^{-1} h_{0}\right)=1 \text { but } x\left(h_{0}\right)=0
$$


and so $g \cdot x \neq x$, that is, $x \in O(g)$. Hence it follows that $O \cap O(g) \neq \emptyset$ for all non-empty open subsets $O$. So, $O(g)$ is dense in $2^{\mathbb{F}_{2}}$. By the Baire category theorem, $\bigcap_{g \in \mathbb{F}_{2} \backslash\{e\}} O(g)$ is also dense in $2^{\mathbb{F}_{2}}$.

Since for each $g, h \in \mathbb{F}_{2} \backslash\{e\}, h \cdot O(g)=O\left(h g h^{-1}\right)$,

$\bigcap_{g \in \mathbb{F}_{2} \backslash\{e\}} O(g)$ is $\mathbb{F}_{2}$-invariant.

Let $X=\bigcap_{g \in \mathbb{F}_{2} \backslash\{e\}} O(g)$. Then $X$ is a dense $G_{\delta}$ subset of the perfect Polish space $2^{\mathbb{F}_{2}}$. So $X$ is a perfect Polish space. Also $X$ is an $\mathbb{F}_{2}$-invariant subset of $2^{\mathbb{F}_{2}}$ such that for any $x \in X, g \cdot x \neq x$ for every $g \in \mathbb{F}_{2} \backslash\{e\}$. In other words, the action of $\mathbb{F}_{2}$ is topologically free on $X$.

Lemma 2 There exists $x_{0} \in 2^{\mathbb{F}_{2}}$ such that the orbit $\left\{\alpha_{g}\left(x_{0}\right): g \in \mathbb{F}_{2}\right\}$ is free and the suborbit $\left\{a^{n} \cdot x_{0}: n \in \mathbb{Z}\right\}$ is dense in $2^{\mathbb{F}_{2}}$.

Proof. Since the action on $X$ is topologically free, for any $x_{1} \in X$, the orbit $\left\{g \cdot x_{1}: g \in \mathbb{F}_{2}\right\}$ is free.

Take any pair of basic clopen subsets of $2^{\mathbb{F}_{2}}$. Call them $W_{1}$ and $W_{2}$ with

$W_{1}=\left\{x \in 2^{\mathbb{F}_{2}}: x(g)=i_{g}\right.$ for $\left.g \in S_{1}\right\}$ and $W_{2}=\left\{y \in 2^{\mathbb{F}_{2}}: y(g)=j_{g}\right.$ for $\left.g \in S_{2}\right\}$

where $S_{1}$ and $S_{2}$ are non-empty finite subsets of $\mathbb{F}_{2}$ and $i_{g} \in\{0,1\}$ for $g \in S_{1}$ and $j_{g} \in\{0,1\}$ for $g \in S_{2}$. Since $S_{2} S_{1}^{-1}$ is a finite set and $\langle a\rangle=\left\{a^{m}: m \in \mathbb{Z}\right\}$ (subgroup of $\mathbb{F}_{2}$ ) is infinite, there exists $k_{0} \in \mathbb{N}$ such that $a^{n} \notin S_{2} S_{1}^{-1}$ for $|n|>k_{0}$, that is, $a^{n} S_{1} \cap S_{2}=\emptyset$.

Let $y \in 2^{\mathbb{F}_{2}}$ be defined by

$$
y(h)=\left\{\begin{array}{ll}
i_{a^{-n} h} & \text { when } h \in a^{n} S_{1} \\
j_{h} & \text { when } h \in S_{2} \\
1 & \text { otherwise }
\end{array} .\right.
$$

Then $y \in W_{2}$. Suppose $h \in S_{1}$. Then $a^{n} h \in a^{n} S_{1}$. Since

$$
\left(a^{-n} \cdot y\right)(h)=y\left(a^{n} h\right)=i_{a^{-n} a^{n} h}=i_{h},
$$

it follows that $a^{-n} \cdot y \in W_{1}$, which implies that $y \in a^{n} \cdot W_{1}$.

Hence $\left(a^{n} \cdot W_{1}\right) \cap W_{2}$ is a non-empty clopen subset of $2^{\mathbb{F}_{2}}$ for $|n|>k_{0}$.

This definition of $y$ makes sense because $a^{n} S_{1} \cap S_{2}=\emptyset$. Since $X$ is a dense subset of $2^{\mathbb{F}_{2}}$ it follows that

$$
\left(a^{n} \cdot\left(X \cap W_{1}\right)\right) \cap\left(X \cap W_{2}\right)=X \cap\left(\left(a^{n} \cdot W_{1}\right) \cap W_{2}\right) \neq \emptyset .
$$

Let $\mathcal{W}$ be the colllection of all sets of the form $W_{1}$. Then $\mathcal{W}$ is a countable base for the topology of $2^{\mathbb{F}_{2}}$. We remark that each $W \in \mathcal{W}$ is clopen.

Then $\mathcal{V}=\{W \cap X: W \in \mathcal{W}\}$ is a base for the topology of $X$. Then $\mathcal{V}$ is a countable base, of non-empty clopen sets. Let $\left(V_{1}, V_{2}, \cdots\right)$ be an enumeration of $\mathcal{V}$.

Since $X$ is Polish it may be regarded as a separable metric space (with complete metric $d$ ). Let $D$ be a countable dense subset of $X$. Let $\mathcal{B}$ be the 
collection of all open balls with rational radius and centred on points of $D$. Then $\mathcal{B}$ is a countable base for the topology of $X$.

Given any $\varepsilon>0$ and any $V \in \mathcal{V}$ there is $B \in \mathcal{B}$ such that $B \subset V$ and the diameter of $B$ is less than $\varepsilon$. Since $\mathcal{V}$ is also a base for the topology there is $U \in \mathcal{V}$ with $U \subset B$. So diameter $U$ is less than $\epsilon$ and $U \subset V$.

We shall now obtain a sequence $\left(U_{n}\right)$ in $\mathcal{V}$ such that

(i) for each $n, a^{-m(n)} \cdot U_{n} \subset V_{n}$ for some $m(n) \in \mathbb{N}$,

(ii) diameter $\left(U_{n}\right)<\frac{1}{2^{n}}$ and

(iii) $U_{n} \subset U_{n-1}$ for $n>1$.

Take $V_{1}$. Then we can find $B \subset V_{1}$, where $B$ is an open ball of diameter less than $\frac{1}{2}$. Now let $U_{1} \in \mathcal{V}$ be a subset of $B$. Clearly (ii) is satisfied. So also is (i) on putting $m(1)=0$.

Now suppose that $\left(U_{1}, U_{2}, \ldots, U_{n}\right)$ have been constructed.

Now take $V_{n+1}$. Arguing as above, there exists a (clopen) $Z \in \mathcal{V}$ such that $Z \subset U_{n}$ and its diameter is less than $\frac{1}{2^{n+1}}$. Then one has $m \in \mathbb{N}$ such that

$$
\left(a^{m} \cdot V_{n+1}\right) \cap Z \neq \emptyset .
$$

Since $\left(a^{m} \cdot V_{n+1}\right) \cap Z$ is a non-empty open subset, one has a non-empty clopen set $U_{n+1}$ in $\mathcal{V}$ such that

$$
U_{n+1} \subset\left(a^{m} \cdot V_{n+1}\right) \cap Z .
$$

Since $U_{n+1} \subset Z \subset U_{n}$, the diameter of $U_{n+1}$ is less than that of $Z$ which is less than $\frac{1}{2^{n+1}}$ and $U_{n+1} \subset U_{n}$. Also we have $U_{n+1} \subset a^{m} \cdot V_{n+1}$, that is, $a^{-m} \cdot U_{n+1} \subset V_{n+1}$.

We now obtain a sequence in $X$ by choosing $x_{n}$ from each $U_{n}$. Clearly $\left(x_{n}\right)$ is a Cauchy sequence. Since the metric space is complete, the sequence converges to a point $x_{0}$.

We claim $\left\{a^{m} \cdot x_{0}: m \in \mathbb{Z}\right\}$ is dense in $X$.

Take any non-empty open subset $O$ of $X$, one has $V_{n} \in \mathcal{V}$ such that $V_{n} \subset O$. By (i), there exists $m \in \mathbb{Z}$ with $m \geq 0$ such that

$$
a^{m} \cdot U_{n} \subset V_{n} \subset O .
$$

Since $x_{0} \in U_{n}, a^{m} \cdot x_{0} \in O$, which means that $<a>x_{0}$ is dense in $X$. Since $X$ is dense in $2^{\mathbb{F}_{2}}$ so also is $\langle a\rangle x_{0}$.

Corollary 3 (i) There exists $Y$, a dense $G_{\delta}$ subset of $X$, such that, for every $y \in Y$, the sub-orbit $\langle a\rangle y$ is dense in $X$ and hence dense in $2^{\mathbb{F}_{2}}$.

(ii) There exists $Z$, a dense $G_{\delta}$ subset of $2^{\mathbb{F}_{2}}$, such that, for every $z \in Z$, the sub-orbit $\langle a\rangle z$ is dense in $2^{\mathbb{F}_{2}}$. Furthermore we may suppose that $Z$ is invariant under the action of $\mathbb{F}_{2}$.

Proof. (i) This follows from Lemma 1.1 [12]. Alternatively, apply Proposition 6.5.5 and Lemma 6.4.7 [11].

(ii) By the Baire category theorem, the intersection of countably many dense $G_{\delta}$ subsets of $2^{\mathbb{F}_{2}}$, is a dense $G_{\delta}$ subset. Put $Z=\cap\left\{g Y: g \in \mathbb{F}_{2}\right\}$. 
We wish to obtain similar results for actions of the free group on the structure space, $S$, of the Dixmier algebra. We shall do this by applying results on induced actions given in Section 6.4 of [11]. Although this can be done more generally, we shall focus on the Dixmier algebra.

Let $K$ be a perfect Polish space which is compact, for example $2^{\mathbb{F}_{2}}$. Then there is a natural injective $*$-homomorphism $j$ from $C(K)$ into $B(K) / M(K) \cong C(S)$, the Dixmier algebra. Using the familiar duality between commutative (unital) $C^{*}$-algebras and compact Hausdorff spaces, there is a continuous surjection $\rho: S \rightarrow K$ such that $j(f)=f \circ \rho$.

Let $\theta$ be a homeomorphism of $K$ onto $K$. As in the beginning of Section 2, let $h_{\theta}$ be the corresponding $*$-automorphism of $C(K)$. Also $f \longmapsto f \circ \theta$ induces an automorphism $\widehat{h}_{\theta}$ of $B(K) / M(K)$. Since $B(K) / M(K)$ can be identified with $C(S)$, there exists $\widehat{\theta}$ in $\operatorname{Homeo}(S)$ corresponding to $\widehat{h}_{\theta}$. Clearly, $\widehat{h}_{\theta}$ restricts to the automorphism, $h_{\theta}$, of $C(K)$. It can be shown that $\widehat{h}_{\theta}$ is the unique extension of $h_{\theta}$ to a $*$-automorphism of $C(S)$. Then $\theta \rightarrow \widehat{\theta}$ is an injective group homomorphism from $\mathrm{Homeo}(K)$ into $\operatorname{Homeo}(S)$.

Lemma 4 For each $s \in S, \theta(\rho s)=\rho(\widehat{\theta} s)$.

Proof. See Corollary 6.4.3 [11].

To apply this lemma we now put $K=2^{\mathbb{F}_{2}}$ and recall the action, $g \rightarrow \alpha_{g}$, of $\mathbb{F}_{2}$ on $2^{\mathbb{F}_{2}}$. Here this action is an injective group homomorphism of $\mathbb{F}_{2}$ into Homeo $\left(2^{\mathbb{F}_{2}}\right)$. It follows that $g \rightarrow \widehat{\alpha_{g}}$, is an injective group homomorphism of $\mathbb{F}_{2}$ into $\operatorname{Homeo}(S)$. So we can define an induced action $\widehat{\alpha}$ on $S$ by $\widehat{\alpha}_{g}=\widehat{\alpha_{g}}$ for $g \in \mathbb{F}_{2}$.

Proposition 5 There exists $s_{0} \in S$ such that the orbit $\left\{\widehat{\alpha}_{g}\left(s_{0}\right): g \in \mathbb{F}_{2}\right\}$ is free and the suborbit $\left\{a^{n} \cdot s_{0}: n \in \mathbb{Z}\right\}$ is dense in $S$. Here we define $a \cdot s=\widehat{\alpha}_{a} s$.

Proof. Let $x_{0}$ be as in 2. There exists $s_{0}$ in $S$ such that $\rho s_{0}=x_{0}$ because $\rho$ is a surjective map from $S$ onto $2^{\mathbb{F}_{2}}$. By Lemma $4 \theta\left(x_{0}\right)=\rho\left(\widehat{\theta}_{s_{0}}\right)$, whenever $\theta=\alpha_{g}$. It now follows from Proposition 6.4.4 [11] that the sub-orbit $\left\{a^{n} \cdot s_{0}: n \in \mathbb{Z}\right\}$ is dense in $S$.

Also, by Proposition 6.4.5 [11], the orbit $\left\{\widehat{\alpha}_{g}\left(s_{0}\right): g \in \mathbb{F}_{2}\right\}$ is free.

Corollary 6 There exists a $\mathbb{F}_{2}$-invariant $Y$, which is a dense $G_{\delta}$ subset of $S$, such that, for $g \in \mathbb{F}_{2}$ and $g \neq e, \widehat{\alpha}_{g}$ has no fixed point in $Y$. Also $s_{0} \in Y$.

Proof. This follows from Lemma 6.4.7 [11].

By applying Lemma 1.1 (3) [12] (see also Proposition 6.5.5 [11]) we obtain:

Corollary 7 There exists a $\mathbb{F}_{2}$-invariant $Z$, which is a dense $G_{\delta}$ subset of $S$, such that, for $g \in \mathbb{F}_{2}$ and $g \neq e, \widehat{\alpha}_{g}$ has no fixed point in $Z$. Also for $y \in Z$, the sub-orbit $\left\{a^{n} \cdot y: n \in \mathbb{Z}\right\}$ is dense in $Z$. 


\section{CONCLUSIONS}

In Lemma 1 we replace $T$ by $S$ and observe that Proposition 5 implies that we can suppose the dense sub-orbit hypothesis is satisfied. This gives:

Proposition 8 Let $\phi$ be a positive linear map from $\ell^{\infty}\left(\mathbb{F}_{2}\right)$ to $J[C(S)]$ such that

$$
\gamma^{g} \circ \phi=\phi \circ \gamma^{g}
$$

for each $g \in \mathbb{F}_{2}$. Then the linear map $\phi$ is identically zero.

In the above, the action $g \rightarrow \gamma^{g}$ of $\mathbb{F}_{2}$ on $J[C(S)]$ is free and ergodic. To see this we can argue as follows.

Take $g \in G$ with $g \neq e$, the neutral element of $G$. If $\gamma^{g}$ were not properly outer, one would have a $G$-invariant non-empty clopen subset $E$ of $S$ such that $\left.\gamma^{g}\right|_{C(S) \chi_{E}}$ is the identity. Then we would have $\gamma^{g} J \chi_{E} J f=J \chi_{E} J f$ for all $f \in C(S)$ which means that

$$
\left(\chi_{E} f\right)\left(\alpha_{g^{-1}} \alpha_{h} s_{0}\right)=\chi_{E} f\left(\alpha_{h} s_{0}\right) \text { for all } f \in C(S) \text { and } h \in G .
$$

Since $G s_{0}$ is dense and $E$ is non-empty clopen, one has $h \in G$ such that $h s_{0} \in E$. Since $\alpha_{g^{-1}} \alpha_{h} s_{0} \neq \alpha_{h} s_{0}$, one has $f \in C(S)$ such that $f\left(\alpha_{g^{-1}} \alpha_{h} s_{0}\right) \neq f\left(\alpha_{h} s_{0}\right)$. Since $\alpha_{h} s_{0} \in E, \alpha_{g^{-1}} \alpha_{h} s_{0} \in E$ and so we have $f\left(\alpha_{g^{-1}} \alpha_{h} s_{0}\right)=f\left(\alpha_{h} s_{0}\right)$. But this would be a contradiction. So, the action $g \mapsto \gamma^{g}$ is free on $J[C(S)]$. Next take any $f \in C(S)$ and suppose $\gamma^{g}(J f)=J f$ for all $g \in G$. Then we have $f\left(\alpha_{g^{-1}} \alpha_{h} s_{0}\right)=f\left(\alpha_{h} s_{0}\right)$ for all $g, h \in G$ and so in particular, $f\left(\alpha_{h} s_{0}\right)=f\left(s_{0}\right)$ for all $h \in G$. Since $G s_{0}$ is dense in $S$ and $f$ is continuous, it follows that $f(s)=f\left(s_{0}\right)$ for all $s \in S$ and so $f=f\left(s_{0}\right) 1$. So, the action $\gamma$ is ergodic.

Let $G$ be a countably infinite group which acts freely and ergodically as *-automorphisms of $C(S)$. Then there exists a corresponding monotone crossproduct $\mathrm{C}^{*}$-algebra $M(C(S), G)$ which is a wild factor. Then Theorem 3.4 [12] tells us that $M(C(S), G)$ is isomorphic to the Takenouchi-Dyer factor, for every choice of $G$, provided the action is free and ergodic. It is also isomorphic to $M\left(C(S), \oplus \mathbb{Z}_{2}\right)$. In particular $M\left(C(S), \mathbb{F}_{2}\right) \cong M\left(C(S), \oplus \mathbb{Z}_{2}\right)$. Each of these monotone cross-products corresponds to a hyperfinite Borel equivalence relation on a perfect Polish space (see [12]). See Example 6.1.4 [11]. For more details and generalisations, see Chapter 7 [11]. We have that the factor $M\left(C(S), \mathbb{F}_{2}\right)$ is hyperfinite. We shall show that it is not an injective $\mathrm{C}^{*}$-algebra. (For monotone cross-products see $[9,10,5,11,12,13])$.

Lemma 9 Let $A$ and $B$ be commutative monotone complete $C^{*}$-algebras. Let $A$ be embedded as a $C^{*}$-subalgebra of $B$. Let $G$ be a countable group of *automorphisms of $B$, each of which maps $A$ into A. Let $M(A, G)$ be injective. Then there exists a positive linear projection (conditional expectation) $\Phi$ from $B$ onto $A$ with the following properties. For each $g \in G$, and $x \in B$,

$$
\Phi g(x)=g \Phi(x) .
$$

Proof. This follows from Proposition 2.3 [18]. 
Theorem 10 The hyperfiniteTakenouchi-Dyer factor is not injective.

Proof. $\{$ sketch $\}$ In Lemma 9 put $B=\ell^{\infty}\left(\mathbb{F}_{2}\right)$ and put $A=J[C(S)]$.

Asssume that $M\left(C(S), \mathbb{F}_{2}\right)$ is an injective $\mathrm{C}^{*}$-algebra. Then, by Lemma 9 , there exists an equivariant positive linear projection from $B$ onto $A$. Then, by Proposition $8, \Phi$ vanishes on $B$. But this is impossible since $\Phi 1=1$.

\section{References}

[1] L.J. Bunce and J.D.M. Wright, On Sullivan's invariant measure problem, Proc. Amer. Math.Soc., 103(1988), 870-874.

[2] A. Connes, Classification of injective factors, Ann. Math., 104(1976), 73115.

[3] R. Dougherty and M. Forman, Banach-Tarski decomposition using sets with the property of Baire, J. Amer. Math. Soc., 7(1994), 75-124.

[4] J.A. Dyer, Concerning $A W^{*}$-algebras, Not. Amer. Math. Soc., 17(1970), 788.

[5] M. Hamana, Tensor products for monotone complete $\mathrm{C}^{*}$-algebras II, Jpn. J. Math., 8(1982) 285-295.

[6] G. Hjorth, Classification and Orbit Equivalence Relations, Mathematical Surveys and Monographs, American Mathematical Society, 75(2000)

[7] G. Hjorth and A.S. Kechris, Rigidity theorems for actions of product groups and countable Borel equivalence relations, Memoirs Amer. Math. Soc., 177(2005), 1-109.

[8] E.C. Lance, Recent developements in the theory of von Neumann algebras May 1981, Preface to the English Edition: J. Dixmier, Von Neumann Algebras, North-Holland Mathematical Library.

[9] K. Saitô, $A W^{*}$-algebras with monotone convergence propery and type III, non $W^{*}, A W^{*}$-factors, Lecture Notes in Mathematics, vol 650,131134,Springer, Berlin, Heidelberg, New York, 1978.

[10] K. Saitô, $A W^{*}$-algebras with monotone convergence property and examples by Takenouchi and Dyer, Tohoku Math. J., 31(1979), 31-40.

[11] K. Saitô and JDM Wright, Monotone complete $C^{*}$-algebras and generic dynamics, Springer Monographs in Mathematics, 2015.

[12] D. Sullivan, B. Weiss and JDM Wright, Generic dynamics and monotone complete $C^{*}$-algebras, Trans. Amer. Math. Soc., 295(1986), 795-809.

[13] O. Takenouchi, A non- $W^{*}, A W^{*}$-factor, Lecture Notes in Mathematics, vol 650, 135-139, Springer, Berlin, Heidelberg, New York 1978. 
[14] M. Takesaki, Theory of Operator algebras III, Springer, Berlin, Heidelberg, New York 2001.

[15] S. Wagon, The Banach-Tarski Paradox, Cambridge University Press, Cambridge 1987 .

[16] J.D.M. Wright, On semi-finite $A W^{*}$-algebras, Math Proc. Camb. Philos. Soc., 79(1976), 443-446.

[17] J.D.M. Wright, Hyperfiniteness in wild factors, J. London Math. Soc., 28(1988), 492-502.

[18] J.D.M. Wright, Paradoxical decomposition of the cube and injectivity, Bull. London Math. Soc., 22(1989), 18-24.3 